\title{
Scale-free collaborative protocol design for state and regulated state synchronization of multi-agent systems with arbitrary fast convergence ${ }^{\text {i⿱ }}$
}

\author{
Zhenwei Liu $^{\mathrm{a}, *}$, Ali Saberi ${ }^{\mathrm{b}}$, Anton A. Stoorvogel ${ }^{\mathrm{c}}$, \\ Donya Nojavanzadeh ${ }^{\mathrm{b}}$ \\ ${ }^{a}$ College of Information Science and Engineering, Northeastern University, Shenyang, China \\ ${ }^{\mathrm{b}}$ School of Electrical Engineering and Computer Science, Washington State University, Pullman, WA, USA \\ ${ }^{\mathrm{c}}$ Department of Electrical Engineering, Mathematics and Computer Science, University of Twente, Enschede, the \\ Netherlands
}

Received 5 October 2020; received in revised form 1 March 2021; accepted 3 April 2021

Available online 20 April 2021

\begin{abstract}
In this paper, we design scale-free collaborative protocols for state and regulated state synchronization of homogeneous multi-agent systems (MAS) with arbitrary fast convergence. The protocol design solely depends on the knowledge of the agents' model and does not require any information about the communication network and the number of agents. Moreover, our protocols can achieve synchronization with any desired convergence rate by simply tuning a design parameter.
\end{abstract}

(C) 2021 The Franklin Institute. Published by Elsevier Ltd. All rights reserved.

\footnotetext{
is This work is supported by the Nature Science Foundation of Liaoning Province, PR China under Grant 2019MS-116 and 2019-KF-03-02, the Fundamental Research Funds for the Central Universities of China under Grant N2004014, and the United States National Science Foundation under Grant 1635184.

* Corresponding author.

E-mail addresses: liuzhenwei@ise.neu.edu.cn (Z. Liu), saberi@wsu.edu (A. Saberi), a.a.stoorvogel@utwente.nl (A.A. Stoorvogel), donya.nojavanzadeh@wsu.edu (D. Nojavanzadeh).
} 


\section{Introduction}

Synchronization or consensus problem of multi-agent systems has attracted much more attention in recent years due to widely practical applications in cooperative control of MAS such as autonomous vehicles, satellites/robots system, distributed sensor network, and others, see Refs. [1-6] and references therein. The objective of synchronization of MAS is to secure an asymptotic agreement on a common state or output trajectory by local interaction among agents. It is worthwhile to note that state synchronization inherently requires homogeneous MAS.

Most of the proposed protocols in the literature for synchronization of MAS requires some knowledge of the communication network such as bounds on the spectrum of the associated Laplacian matrix or the number of agents. As it is pointed out in [7-9], these protocols suffer from scale fragility wherein stability properties are lost for large-scale networks or when the communication graph changes. Therefore, there exist a current research effort focusing on scalability of consensus laws. Recently, we have introduced a new generation of scale-free protocols for synchronization and almost synchronization of MAS, see [10,11], where the agents are subject to input saturation and external disturbances, respectively. The scale-free framework, utilizing localized information exchange among the neighbors does not require any knowledge of the communication topology and the size of the network.

In practical applications, one of the key elements in designing consensus protocols is performance. The asymptotic convergence rate is defined in [12] as an indicator for the performance which we will use in this paper. The convergence rate is an important element in designing protocols. Typically, the communication topology through which the agents communicate is a deciding factor in establishing the convergence rate of the protocol. More explicitly, the convergence rate of various existing protocols for continuous-time MAS with undirected communication graphs depends on the second smallest eigenvalue of Laplacian matrix of the associated communication graph, also known as the algebraic connectivity of the graph. In discrete-time MAS, the largest modulus of the eigenvalues of Perron matrix of the associated communication graph plays the same role in the asymptotic convergence rate, see for example [12-14]. In fact, for a certain class of undirected graphs the algebraic connectivity decreases with an increase in network size, the recent thesis [15] describes this effect and covers some non-exhaustive classes of graphs. In [5, chapter 16], a similar conclusion has been drawn for a directed circulant graph. Therefore, the main goal of this paper is to propose scalable protocols such that the performance of the system is not degraded by increasing the size of the network.

Our main goal in this paper is to develop a new class of scale-free collaborative protocols (parameterized in scalar positive parameter $\mu$ ) to achieve state and regulated state synchronization of MAS with arbitrary fast convergence. Our proposed protocols have the following properties.

- For any positive value of parameter $\mu$, state and regulated state synchronizations are achieved for a MAS with any size of the network and with any communication topology.

- The proposed protocols achieve any given desired convergence rate by suitably choosing parameter $\mu$. In fact, by increasing $\mu$, we can achieve any a priori given asymptotic convergence rate.

- The structure of the proposed protocols is independent of the parameter $\mu$; thus, one may develop the structure at one stage and tune the parameter $\mu$ later so as to obtain 
the desired convergence rate. Due to continuity in $\mu$, tuning may be even carried out online. Hence, the method is a one-shot design and is not iterative.

\subsection{Notations and definitions}

For a square matrix $M \in \mathbb{R}^{n \times n}$, we will denote the set of eigenvalues of $M$ by $\lambda(M)$ while $\rho(M)$ denotes the spectral radius of $M$. A matrix $M$ is Hurwitz stable if all its eigenvalues are in the open left half complex plane. $A \otimes B$ denotes the Kronecker product between $A$ and B.

The communication network can be connected to a weighted graph $\mathcal{G}$ to describe the information flow between the agents. A weighted graph $\mathcal{G}$ is defined by a triple $(\mathcal{V}, \mathcal{E}, \mathcal{A})$ where $\mathcal{V}=\{1, \ldots, N\}$ is the node set, $\mathcal{E}$ is a set of pairs of nodes indicating connections among nodes, and $\mathcal{A}=\left[a_{i j}\right] \in \mathbb{R}^{N \times N}$ is the weighted adjacency matrix with non negative elements $a_{i j}$. Each pair in $\mathcal{E}$ is called an edge, where $a_{i j}>0$ denotes an edge $(j, i) \in \mathcal{E}$ from node $j$ to node $i$ with weight $a_{i j}$. If there is no edge from node $j$ to node $i$ then we will have $a_{i j}=0$. We assume there are no self-loops, i.e. we have $a_{i i}=0$. A path from node $i_{1}$ to $i_{k}$ is a sequence of nodes $\left\{i_{1}, \ldots, i_{k}\right\}$ such that $\left(i_{j}, i_{j+1}\right) \in \mathcal{E}$ for $j=1, \ldots, k-1$. A directed tree is a subgraph (subset of nodes and edges) in which every node has exactly one parent node except for one node, called the root, which has no parent node. A directed spanning tree is a subgraph which is a directed tree containing all the nodes of the original graph. If a directed spanning tree exists, the root has a directed path to every other node in the tree, see [16].

For a weighted graph $\mathcal{G}$, the matrix $L=\left[\ell_{i j}\right]$ with

$\ell_{i j}=\left\{\begin{array}{cc}\sum_{k=1}^{N} a_{i k}, & i=j, \\ -a_{i j}, & i \neq j,\end{array}\right.$

is called the Laplacian matrix associated with the graph $\mathcal{G}$.

The asymptotic convergence rate (convergence factor) is an important concept to measure the performance of a MAS. The asymptotic convergence rate of a stable linear system

$\dot{x}(t)=M x(t), \quad(M$ is Hurwitz stable $)$,

with $x \in \mathbb{R}^{n}$, is defined in [12] and [6] as

$r_{\text {asym }}=\max _{x(0) \neq 0} \lim _{t \rightarrow \infty}\left(\frac{\|x(t)\|}{\|x(0)\|}\right)^{\frac{1}{t}}$

and the associated convergence time is defined as

$\tau_{\text {asym }}=\frac{-1}{\log \left(r_{\text {asym }}\right)}$.

We have

$\max _{x(0) \neq 0} \lim _{t \rightarrow \infty}\left(\frac{\|x(t)\|}{\|x(0)\|}\right)^{\frac{1}{t}}=\lim _{t \rightarrow \infty}\left(\left\|e^{M t}\right\|\right)^{\frac{1}{t}}=\rho\left(e^{M}\right)$

where we used Gelfands spectral radius formula [17] that connects the norm and the spectral radius $\rho(\cdot)$ of a matrix. Hence

$r_{\text {asym }}=\rho\left(e^{M}\right)=\max _{i}\left(\left\|e^{\operatorname{Re}\left(\lambda_{i}(M)\right)}\right\|\right)=\max _{i}\left(e^{\operatorname{Re}\left(\lambda_{i}(M)\right)}\right)=e^{\operatorname{Re}\left(\lambda_{1}(M)\right)}$ 
where $\lambda_{i}(M)$ denotes the $i$ th eigenvalue of $M$ where the eigenvalues of $M$ ordered sequentially by their real part, i.e.

$\operatorname{Re} \lambda_{n}(M) \leq \cdots \leq \operatorname{Re} \lambda_{2}(M) \leq \operatorname{Re} \lambda_{1}(M)<0$.

\section{Problem formulation}

In this paper we consider a MAS consisting of $N$ identical linear agents

$\dot{x}_{i}=A x_{i}+B u_{i}$,

$y_{i}=C x_{i}$,

where $x_{i} \in \mathbb{R}^{n}, u_{i} \in \mathbb{R}^{m}$ and $y_{i} \in \mathbb{R}^{p}$ are the state, input, output of agent $i$ for $i=1, \ldots, N$.

The communication network is composed of $N$ linear combinations and each combination includes agent's own output relative to that of other agents, which is shown as follows for agent $i$

$\zeta_{i}=\sum_{j=1}^{N} a_{i j}\left(y_{i}-y_{j}\right)$

where $a_{i j}>0$ and $a_{i i}=0$. Here we use a weighted and directed graph $\mathcal{G}$ to describe the communication topology of the network, where the nodes of network correspond to the agents and the weight of edges given by the coefficient $a_{i j}$. In the matter of the coefficients of the associated Laplacian matrix $L=\left[\ell_{i j}\right]_{N \times N}, \zeta_{i}$ can be rewritten as

$\zeta_{i}=\sum_{j=1}^{N} \ell_{i j} y_{j}$.

We refer to Eq. (5) as partial-state coupling since only part of the states are communicated over the network. When $C=I$, we call it full-state coupling.

We also introduce a localized information exchange among agents. It means that each agent $i \in\{1, \ldots, N\}$ has access to localized information, denoted by $\hat{\zeta}_{i}$, of the form

$\hat{\zeta}_{i}=\sum_{j=1}^{N} a_{i j}\left(\eta_{i}-\eta_{j}\right)$

where $\eta_{i}$ is a variable produced internally by agent $i$ which will be appropriately chosen in the coming sections.

Our goal is to achieve state and regulated state synchronization among the agents where state synchronization means the state of agents converge asymptotically to a common trajectory, i.e.,

$\lim _{t \rightarrow \infty}\left(x_{i}(t)-x_{j}(t)\right)=0$

for all $i, j \in\{1, \ldots, N\}$, and the regulated state synchronization means the state of agents converge asymptotically to a priory given trajectory $x_{r}$, i.e.,

$\lim _{t \rightarrow \infty}\left(x_{i}(t)-x_{r}(t)\right)=0$,

where $x_{r}$ is generated by a so-called exosystem

$\dot{x}_{r}=A x_{r}, \quad x_{r}(0)=x_{r 0}$,

$y_{r}=C x_{r}$, 
where $x_{r} \in \mathbb{R}^{n}$ and $y_{r} \in \mathbb{R}^{p}$.

Remark 1. It is worth noting that there also exist some other synchronization results, such as event-triggered fault tolerant synchronization [18-20] and fixed-time synchronization [21,22].

To guarantee that each agent gets the information from the exosystem, a nonempty subset $\mathscr{C}$ of the agents is assumed which allows agents have access to their output relative to the output of the exosystem. It means that each agent $i$ has access to the quantity

$\Psi_{i}=\iota_{i}\left(y_{i}-y_{r}\right), \quad \iota_{i}= \begin{cases}1, & i \in \mathscr{C}, \\ 0, & i \notin \mathscr{C} .\end{cases}$

Thus, the information exchange among agents with link of exosystem is given by

$\tilde{\zeta}_{i}=\sum_{j=1}^{N} a_{i j}\left(y_{i}-y_{j}\right)+\iota_{i}\left(y_{i}-y_{r}\right)$.

We can rewrite $\tilde{\zeta}_{i}$ in terms of the coefficients of a so-called expanded Laplacian matrix $\tilde{L}=L+\operatorname{diag}\left\{\iota_{i}\right\}=\left[\tilde{\ell}_{i j}\right]_{N \times N}$ as

$\tilde{\zeta}_{i}=\sum_{j=1}^{N} \tilde{\ell}_{i j}\left(y_{j}-y_{r}\right)$.

where (12) can be written as

$\tilde{\zeta}_{i}=\sum_{j=1}^{N} \tilde{\ell}_{i j}\left(x_{j}-x_{r}\right)$

for the case of full-state coupling. Note that $\tilde{L}$ is not a regular Laplacian matrix associated with a graph since the sum of its rows need not be zero. According to [23, Lemma 7], one can show that all the eigenvalues of $\tilde{L}$ have positive real parts. In particular, the matrix $\tilde{L}$ is invertible.

We need the following definition to explicitly state our problem formulations.

Definition 1. We define the following sets.

1. $\mathbb{G}^{N}$ denotes the set of directed graphs of $N$ agents which contains a directed spanning tree.

2. Given a node set $\mathscr{C}$, we denote by $\mathbb{G}_{\mathscr{C}}^{N}$ the set of all graphs with $N$ nodes containing the node set $\mathscr{C}$, such that every node of the network graph $\mathcal{G} \in \mathbb{G}_{\mathscr{C}}^{N}$ is a member of a directed tree which has its root contained in the node set $\mathscr{C}$. We will refer to the node set $\mathscr{C}$ as root set. Note that a graph $\mathcal{G} \in \mathbb{G}_{\mathscr{C}}^{N}$ does not necessarily contain a directed spanning tree.

Remark 2. We order the set of eigenvalues of Laplacian matrix $L$ associated to the graph $\mathcal{G} \in \mathbb{G}^{N}$ sequentially in an ascending order by the real parts as (see $\left.[3,16]\right)$

$0=\bar{\lambda}_{1}<\operatorname{Re} \bar{\lambda}_{2} \leq \cdots \leq \operatorname{Re} \bar{\lambda}_{n}$.

Now we formulate the problems of scalable state synchronization. 
Problem 1. Consider a MAS (3) with associated network communication (5) and a given desired convergence rate $r_{\text {asym }}^{*}$.

The problem of scalable state synchronization with arbitrarily fast convergence utilizing collaborative protocols is to find, if possible, a parameterized linear dynamic protocol in scalar $\mu>0$, using only knowledge of the agent models, i.e. $(A, B, C)$ and $r_{\text {asym }}^{*}$, of the form:

$\dot{x}_{i, c}=A_{c}^{\mu} x_{i, c}+B_{c}^{\mu} \zeta_{i}+C_{c}^{\mu} \hat{\zeta}_{i}$

$u_{i}=F_{c}^{\mu} x_{i, c}$

where $\hat{\zeta}_{i}$ is defined in Eq. (6) with $\eta_{i}=M_{c} x_{i, c}$, and $x_{i, c} \in \mathbb{R}^{n_{c}}$, such that

- the protocol achieves state synchronization (7) for any $\mu>0$, any $N$ and any graph $\mathscr{G} \in \mathbb{G}^{N}$ where $\mathbb{G}^{N}$ is defined in Definition 1 .

- for a given desired $r_{\text {asym }}^{*}$ and any given graph $\mathscr{G} \in \mathbb{G}^{N}$, by choosing parameter $\mu$ sufficiently large, the protocol achieves a convergence rate (for the synchronization error) less than $r_{\text {asym. }}^{*}$.

Next we formulate the problem of scalable regulated state synchronization.

Problem 2. Consider a MAS (3) with associated network communication (12) and associated exosystem (9) and a given desired convergence rate $r_{\text {asym }}^{*}$.

The problem of scalable regulated state synchronization with arbitrarily fast convergence utilizing collaborative protocols is to find, if possible, a parameterized linear dynamic protocol in scalar $\mu>0$, for each agent, using only knowledge of the agent models, i.e. $(A, B, C)$ and $r_{\text {asym }}^{*}$, of the form:

$\dot{x}_{i, c}=A_{c}^{\mu} x_{i, c}+B_{c}^{\mu} \tilde{\zeta}_{i}+C_{c}^{\mu} \hat{\zeta}_{i}$

$u_{i}=F_{c}^{\mu} x_{i, c}$,

where $\hat{\zeta}_{i}$ is defined in Eq. (6) with $\eta_{i}=M_{c} x_{i, c}$, and $x_{i, c} \in \mathbb{R}^{n_{c}}$, such that

- the protocol achieves regulated state synchronization (8) for any $\mu>0$, any $N$ and any graph $\mathscr{G} \in \mathbb{G}_{\mathscr{C}}^{N}$ where $\mathbb{G}_{\mathscr{C}}^{N}$ is defined as Definition 1.

- for a given desired $r_{\text {asym }}^{*}$, any given graph $\mathscr{G} \in \mathbb{G}_{\mathscr{C}}^{N}$ and any $x_{r}$, by choosing parameter $\mu$ sufficiently large, the protocol achieves a convergence rate (for the synchronization error) less than $r_{\text {asym }}^{*}$.

Remark 3. We refer to Problems 1 and 2 as scalable state and regulated state synchronization with arbitrarily fast convergence problem utilizing collaborative protocols for MAS via fullstate coupling, respectively, when $y_{i}=x_{i}$ (i.e. when matrix $C=I$ ).

Remark 4. It is worth noting that the formulations in Problems 1 and 2 do not specify how the matrices of the protocols should evolve with parameter $\mu$. However, our designs, as will be given in this paper, provide an explicit solution from which the protocol matrices can be derived. The structure of the proposed protocols is independent of the parameter $\mu$; thus, one may develop the structure of the protocol first and tune the parameter $\mu$ later so as to obtain the desired convergence rate. Due to continuity in $\mu$, tuning may be even carried out online. Hence, the method is a one-shot design and is not iterative. 


\section{Protocol design for state synchronization}

In this section, we design scale-free protocols to achieve state synchronization with desired convergence rate. We make the following assumption on agent models.

Assumption 1. We assume that $(A, B)$ is stabilizable and $(A, C)$ is detectable. Moreover, agents are at most weakly unstable, i.e. all eigenvalues of $A$ are in the closed left half complex plane.

Remark 5. Assumption 1 requires the dynamic of system is weakly unstable. Note that weakly unstable systems can have repeated poles on $j \omega$ axis as such they include agents that are polynomially unstable such as double-integrators or chain of integrators. We would like to point out that in most of practical applications, such as multi-robot systems and micro-grid systems with distributed generators and etc, agents are weakly unstable.

\subsection{Full-state coupling}

We consider the following protocol.

Then, we have the following theorem.

Theorem 1. Consider a MAS consisting of $N$ agents (3) with $C=I$ satisfying Assumption 1. Let the associated network communication be given by (5).

Then, the problem of scalable state synchronization with arbitrarily fast convergence as defined in Problem 1 is solvable. In particular, the parameterized linear dynamic Protocol 1

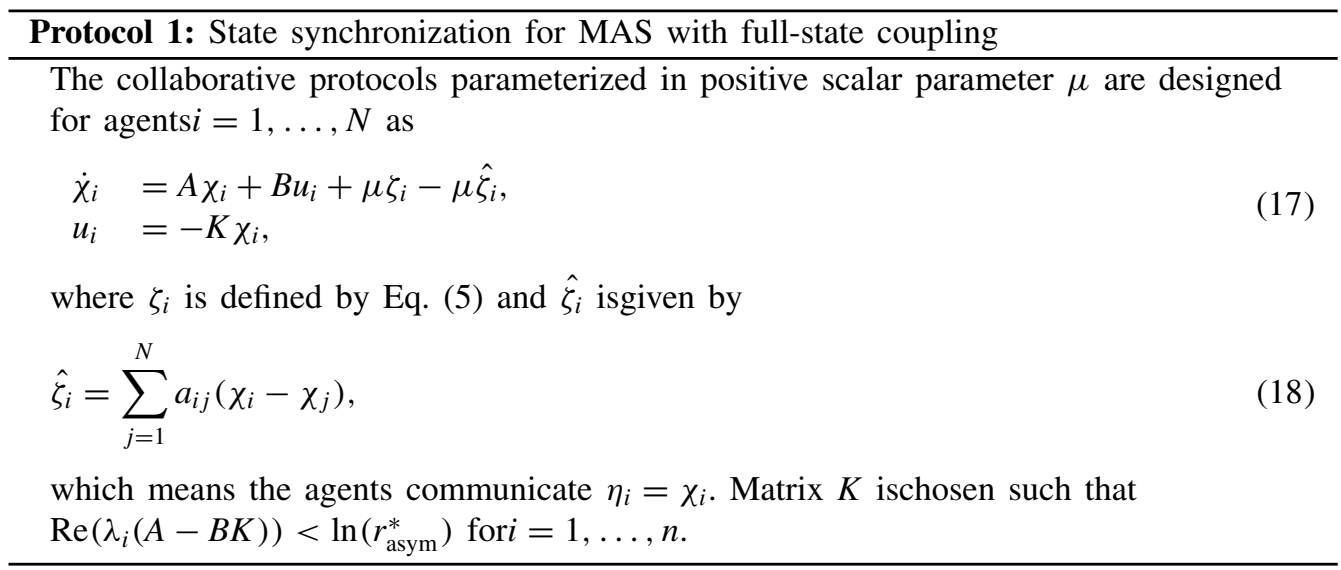

1. achieves state synchronization for any $\mu$, and any graph $\mathcal{G} \in \mathbb{G}^{N}$ with any $N$.

2. for a given desired $r_{\text {asym }}^{*}$ and any given graph $\mathscr{G} \in \mathbb{G}^{N}$ by choosing parameter $\mu$ sufficiently large, the protocol achieves a convergence rate (for the synchronization error) less than $r_{\text {asym. }}^{*}$.

To obtain this result, we need the following lemma for the Laplacian matrix $L$ which its proof is provided in [11, Lemma 1]. 
Lemma 1. Let a Laplacian matrix $L \in \mathbb{R}^{N \times N}$ be given associated with a graph that contains a directed spanning tree. We define $\hat{L} \in \mathbb{R}^{(N-1) \times(N-1)}$ as a matrix $\hat{L}=\left[\hat{\ell}_{i j}\right]$ with $\hat{\ell}_{i j}=\ell_{i j}-\ell_{N j}$.

Then the eigenvalues of $\hat{L}$ are equal to the nonzero eigenvalues of $L$, that is $\lambda(\hat{L})=$ $\left\{\lambda_{2}(L), \lambda_{3}(L), \ldots, \lambda_{N}(L)\right\}$.

Proof of Theorem 1. The proof includes two steps.

Step 1: The proof of state synchronization

Let $\bar{x}_{i}=x_{i}-x_{N}$ and $\bar{\chi}_{i}=\chi_{i}-\chi_{N}$. Then, we have

$$
\begin{aligned}
\dot{\bar{x}}_{i} & =A \bar{x}_{i}-B K \bar{\chi}_{i} \\
\dot{\bar{\chi}}_{i} & =(A-B K) \bar{\chi}_{i}+\mu \sum_{j=1}^{N-1} \hat{\ell}_{i j} \bar{x}_{j}-\mu \sum_{j=1}^{N-1} \hat{\ell}_{i j} \bar{\chi}_{j}
\end{aligned}
$$

where $\hat{\ell}_{i j}=\ell_{i j}-\ell_{N j}$. Then, by defining

$\bar{x}=\left(\begin{array}{lll}\bar{x}_{1}^{\mathrm{T}} & \ldots & \bar{x}_{N-1}^{\mathrm{T}}\end{array}\right)^{\mathrm{T}}, \quad \bar{\chi}=\left(\begin{array}{lll}\bar{\chi}_{1}^{\mathrm{T}} & \ldots & \bar{\chi}_{N-1}^{\mathrm{T}}\end{array}\right)^{\mathrm{T}}$

we have the closed-loop system as

$\dot{\bar{x}}=(I \otimes A) \bar{x}-(I \otimes B K) \bar{\chi}$

$\dot{\bar{\chi}}=(I \otimes(A-B K)-\mu \hat{L} \otimes I) \bar{\chi}+\mu \hat{L} \otimes I \bar{x}$

where $\hat{L}=\left[\hat{\ell}_{i j}\right]_{(N-1) \times(N-1)}$. By defining $\delta=\bar{x}-\bar{\chi}$, we obtain

$\dot{\bar{x}}=(I \otimes(A-B K)) \bar{x}+(I \otimes B K) \delta$,

$\dot{\delta}=(I \otimes A-\mu \hat{L} \otimes I) \delta$.

From Lemma 1, we know that all eigenvalues of $\hat{L}$ have positive real part. Then, we have

$(S \otimes I)(I \otimes A-\mu \hat{L} \otimes I)\left(S^{-1} \otimes I\right)=I \otimes A-\mu \Lambda \otimes I$

for a non-singular transformation matrix $S$, where $I \otimes A-\mu \Lambda \otimes I$ is upper triangular block matrix with $A-\mu \lambda_{i}(\Lambda) I$ for $i=2, \ldots, N$ on the diagonal blocks and $\Lambda$ is the upper triangular matrix with $\lambda_{i}(\Lambda)=\left\{\lambda_{2}, \lambda_{3}, \ldots, \lambda_{N}\right\}$ on the diagonals. Since all eigenvalues of $A$ are in the closed left half complex plane, $A-\mu \lambda_{i} I$ is a stable matrix. It means that all eigenvalues of $I \otimes A-\mu \hat{L} \otimes I$ have negative real part. Thus, it implies that we just need to prove the stability of

$\dot{\bar{x}}=(I \otimes(A-B K)) \bar{x}$.

Because $A-B K$ is Hurwitz stable, it means that $\bar{x}$ is asymptotically stable, i.e. $\lim _{t \rightarrow \infty} \bar{x}_{i}=$ $x_{i}-x_{N} \rightarrow 0$. Thus, it implies

$x_{i}-x_{j} \rightarrow 0$,

which proves the state synchronization.

Step 2: The proof of arbitrary fast convergence

Let $\phi=\left(\begin{array}{ll}\bar{x}^{\mathrm{T}} & \delta^{\mathrm{T}}\end{array}\right)^{\mathrm{T}}$. From Eq. (20), we have

$\dot{\phi}=A_{F} \phi$ 
with

$A_{F}=\left(\begin{array}{cc}I \otimes(A-B K) & I \otimes B K \\ 0 & I \otimes A-\mu \hat{L} \otimes I\end{array}\right)$

According to the definition of convergence rate (1), the convergence rate of synchronization error dynamics $\phi(t)$ Eq. (22) is

$r_{\text {asym }}=\sup _{\phi\left(t_{0}\right) \neq 0} \lim _{t \rightarrow \infty}\left(\frac{\|\phi(t)\|}{\left\|\phi\left(t_{0}\right)\right\|}\right)^{\frac{1}{t-t_{0}}}=\rho\left(e^{A_{F}}\right)=\max _{i}\left(e^{\operatorname{Re}\left(\lambda_{i}\left(A_{F}\right)\right)}\right)$

where $\lambda_{i}\left(A_{F}\right)$ denotes the $i$ th eigenvalue of $A_{F}$.

Hence, we obtain

$$
\begin{aligned}
r_{\text {asym }} & =\max _{i}\left(e^{\operatorname{Re}\left(\lambda_{i}\left(A_{F}\right)\right)}\right)=\max _{i}\left(e^{\operatorname{Re}\left(\lambda_{i}(A-B K)\right)}, e^{\operatorname{Re}\left(\lambda_{i}(I \otimes A-\mu \hat{L} \otimes I)\right)}\right) \\
& \leq \max _{i}\left(r_{\text {asym }}^{*}, e^{\operatorname{Re}\left(\lambda_{i}(I \otimes A-\mu \hat{L} \otimes I)\right)}\right) \\
& \leq \max _{i}\left(r_{\text {asym }}^{*}, \max _{j}\left(e^{\operatorname{Re}\left(\lambda_{i}\left(A-\mu \bar{\lambda}_{j} I\right)\right)}\right)\right) \\
& \leq \max _{i}\left(r_{\text {asym }}^{*}, e^{\operatorname{Re}\left(\lambda_{i}\left(A-\mu \bar{\lambda}_{2} I\right)\right)}\right) .
\end{aligned}
$$

In the above inequality, we have used the facts that $\operatorname{Re}\left(\lambda_{i}(A-B K)\right)<\ln \left(r_{\text {asym }}^{*}\right), \bar{\lambda}_{j}(j=$ $2, \ldots N)$ are the eigenvalues of $\hat{L}$ which are equal to nonzero eigenvalues of $L$ (see Lemma 1 ) and as such $\bar{\lambda}_{2}$ is the nonzero eigenvalue of $L$ with the smallest real part.

Then, one can choose $\mu$ sufficiently large such that $\operatorname{Re}\left(\lambda_{i}\left(A-\mu \bar{\lambda}_{2} I\right)\right)<\ln \left(r_{\text {asym }}^{*}\right)$. Since we have that all eigenvalue of $A$ are in the closed right half plane, we only choose $-\mu \operatorname{Re}\left(\bar{\lambda}_{2}\right) \leq \ln \left(r_{\text {asym }}^{*}\right)$ so as to

$r_{\text {asym }} \leq r_{\text {asym }}^{*}$

\subsection{Partial-state coupling}

In this section, we propose the Protocol 2 stated in the next page for MAS with partial-state coupling.

Then, we have the following theorem.

Theorem 2. Consider a MAS of $N$ agents (3) satisfying Assumption 1. Let the associated network communication be given by (5).

Then, the problem of scalable state synchronization with arbitrarily fast convergence as defined in Problem 1 is solvable. In particular, the parameterized linear dynamic Protocol 2

- achieves state synchronization for any $\mu$, and any graph $\mathcal{G} \in \mathbb{G}^{N}$ with any $N$.

- for a given desired $r_{\text {asym }}^{*}$ and any given graph $\mathscr{G} \in \mathbb{G}^{N}$ by choosing parameter $\mu$ sufficiently large, the protocol achieves convergence rate (for the synchronization error) less than $r_{\text {asym. }}^{*}$. 


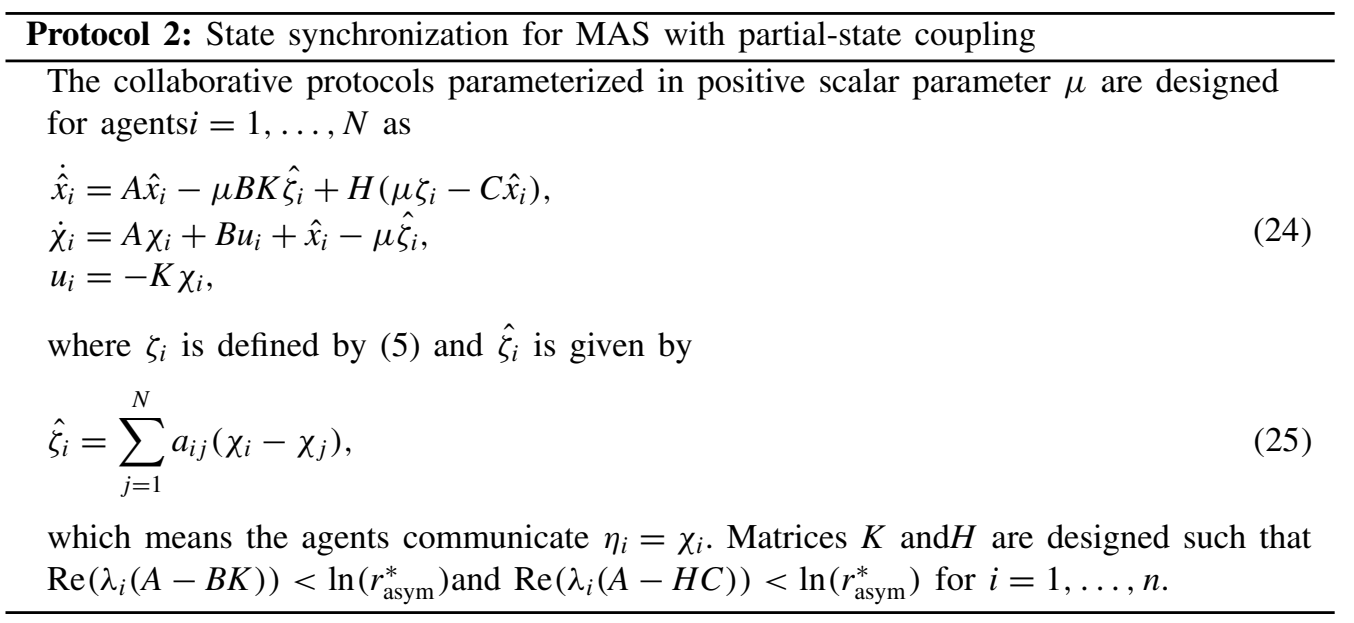

Proof of Theorem 2. Similar to the proof of Theorem 1, we prove the state synchronization first. By defining $\check{x}_{i}=\hat{x}_{i}-\hat{x}_{N}$, we have

$$
\begin{aligned}
\dot{\bar{x}}_{i} & =A \bar{x}_{i}-B K \bar{\chi}_{i} \\
\dot{x}_{i} & =(A-H C) \check{x}_{i}-B K \mu \sum_{j=1}^{N-1} \hat{\ell}_{i j} \bar{\chi}_{j}+\mu H C \sum_{j=1}^{N-1} \hat{\ell}_{i j} \bar{x}_{j} \\
\dot{\bar{\chi}}_{i} & =(A-B K) \bar{\chi}_{i}+\check{x}_{i}-\mu \sum_{j=1}^{N-1} \hat{\ell}_{i j} \bar{\chi}_{j}
\end{aligned}
$$

Following the definition of $\bar{x}$ and $\bar{\chi}$ and by defining

$\check{x}=\left(\begin{array}{lll}\check{x}_{1}^{\mathrm{T}} & \ldots & \check{x}_{N-1}^{\mathrm{T}}\end{array}\right)^{\mathrm{T}}$,

we obtain the closed loop system as

$$
\begin{aligned}
& \dot{\bar{x}}=(I \otimes A) \bar{x}-(I \otimes B K) \bar{\chi} \\
& \dot{\dot{x}}=(I \otimes(A-H C)) \check{x}-\mu(\hat{L} \otimes B K) \bar{\chi}+\mu(\hat{L} \otimes H C) \bar{x} \\
& \dot{\bar{\chi}}=(I \otimes(A-B K)-\mu \hat{L} \otimes I) \bar{\chi}+\check{x}
\end{aligned}
$$

By defining $\delta=\bar{x}-\bar{\chi}$ and $\bar{\delta}=(\mu \tilde{L} \otimes I) \bar{x}-\check{x}$, we have

$$
\begin{aligned}
& \dot{\bar{x}}=(I \otimes(A-B K)) \bar{x}+(I \otimes B K) \delta \\
& \dot{\delta}=(I \otimes A-\mu \hat{L} \otimes I) \delta+\bar{\delta} \\
& \dot{\bar{\delta}}=(I \otimes(A-H C)) \bar{\delta}
\end{aligned}
$$

Similar to the proof of Theorem 1, we know that all eigenvalues of $I \otimes A-\mu \hat{L} \otimes I$ have negative real part. Moreover, since $A-H C$ is Hurwitz stable, we just need to prove the stability of $\dot{\bar{x}}=(I \otimes(A-B K)) \bar{x}$. Furthermore, since $A-B K$ is Hurwitz stable, we have $\bar{x}$ is asymptotically stable, i.e. $\lim _{t \rightarrow \infty} \bar{x}_{i} \rightarrow 0$. Thus, it implies

$x_{i}-x_{j} \rightarrow 0$,

which proves the result.

Next, in the following, we prove the result with arbitrary fast convergence. Let $\bar{\phi}=\left(\begin{array}{lll}\bar{x}^{\mathrm{T}} & \delta^{\mathrm{T}} & \bar{\delta}^{\mathrm{T}}\end{array}\right)^{\mathrm{T}}$ 
then, we obtain

$\dot{\bar{\phi}}=A_{P} \bar{\phi}$

with

$A_{P}=\left(\begin{array}{ccc}I \otimes(A-B K) & I \otimes B K & 0 \\ 0 & I \otimes A-\mu \hat{L} \otimes I & I \\ 0 & 0 & I \otimes(A-H C)\end{array}\right)$.

Then, the asymptotic convergence rate of error dynamics $\bar{\phi}(t)$ would be

$$
\begin{aligned}
r_{\text {asym }} & =\rho\left(e^{A_{P}}\right)=\max _{i}\left(e^{\operatorname{Re}\left(\lambda_{i}\left(A_{P}\right)\right)}\right) \\
& =\max _{i}\left(e^{\operatorname{Re}\left(\lambda_{i}(A-B K)\right)}, e^{\operatorname{Re}\left(\lambda_{i}(I \otimes A-\mu \hat{L} \otimes I)\right)}, e^{\operatorname{Re}\left(\lambda_{i}(A-H C)\right)}\right) \\
& \leq \max _{i}\left(r_{\text {asym }}^{*}, e^{\operatorname{Re}\left(\lambda_{i}(A-\mu \hat{L})\right)}\right) \\
& \leq \max _{i}\left(r_{\text {asym }}^{*}, e^{\operatorname{Re}\left(\lambda_{i}(I \otimes A-\mu \hat{L} \otimes I)\right)}\right) \\
& \leq \max _{i}\left(r_{\text {asym }}^{*}, \max _{j}\left(e^{\operatorname{Re}\left(\lambda_{i}\left(A-\mu \bar{\lambda}_{j} I\right)\right)}\right)\right) \\
& \leq \max _{i}\left(r_{\text {asym }}^{*}, e^{\operatorname{Re}\left(\lambda_{i}\left(A-\mu \bar{\lambda}_{2} I\right)\right)}\right)
\end{aligned}
$$

Here, we used $\operatorname{Re}\left(\lambda_{i}(A-B K)\right)<\ln \left(r_{\text {asym }}^{*}\right), \operatorname{Re}\left(\lambda_{i}(A-H C)\right)<\ln \left(r_{\text {asym }}^{*}\right)$, and $\bar{\lambda}_{j}(j=$ $2, \cdots N)$ are the eigenvalue of $\hat{L}$, where $\bar{\lambda}_{2}$ is the nonzero eigenvalue of $L$ with the smallest real part.

Then, similar to Theorem 1 , by choosing $\mu$ sufficiently large, one can obtain $\operatorname{Re}\left(\lambda_{i}(A-\right.$ $\left.\left.\mu \bar{\lambda}_{2} I\right)\right)<\ln \left(r_{\text {asym }}^{*}\right)$ and hence

$r_{\text {asym }} \leq r_{\text {asym }}^{*}$.

\section{Protocol design for regulated state synchronization}

In this section, we design protocols to achieve regulated state synchronization with desired convergence rate.

\subsection{Full-state coupling}

We propose the Protocol 3 stated in the next page for MAS with full-state coupling.

Then, we have the following theorem.

Theorem 3. Consider a MAS consisting of $N$ agents (3) with $C=I$ satisfying Assumption 1 and the associated exosystem (9). Let the associated network communication be given by Eq. (13).

Then, the problem of scalable regulated state synchronization with arbitrarily fast convergence as defined in Problem 2 is solvable. In particular, the parameterized linear dynamic Protocol 3: 


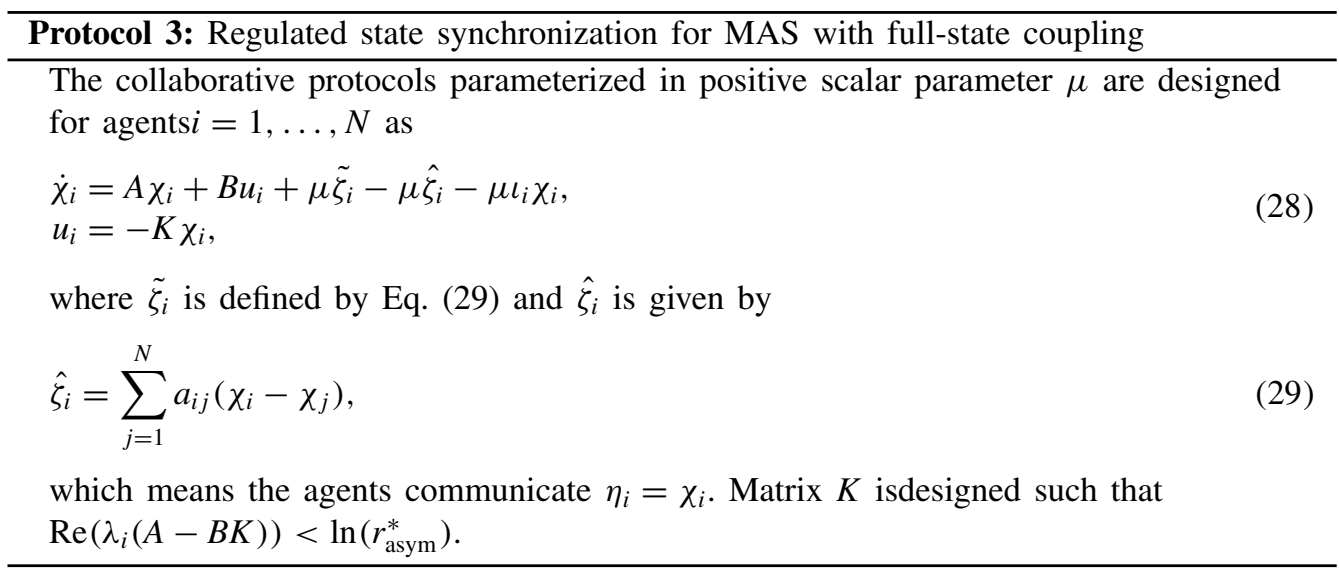

- achieves regulated state synchronization for any $\mu$, and any graph $\mathcal{G} \in \mathbb{G}_{\mathscr{C}}^{N}$ with any $N$.

- for a given desired $r_{\text {asym }}^{*}$, any given graph $\mathscr{G} \in \mathbb{G}_{\mathscr{C}}^{N}$ and any $x_{r}$, by choosing parameter $\mu$ sufficiently large, the protocol achieves convergence rate (for the synchronization error) less than $r_{\mathrm{asym}}^{*}$.

Proof of Theorem 3. Similar to the proof of Theorem 1, the proof consists of two steps.

Step 1: The proof of regulated state synchronization

Let $\tilde{x}_{i}=x_{i}-x_{r}$ and define

$$
\tilde{x}=\left(\begin{array}{lll}
\tilde{x}_{1}^{\mathrm{T}} & \ldots & \tilde{x}_{N}^{\mathrm{T}}
\end{array}\right)^{\mathrm{T}}, \quad \chi=\left(\begin{array}{lll}
\chi_{1}^{\mathrm{T}} & \ldots & \chi_{N}^{\mathrm{T}}
\end{array}\right)^{\mathrm{T}}
$$

then, we obtain the closed loop system as following

$$
\begin{aligned}
& \dot{\tilde{x}}=(I \otimes A) \tilde{x}-(I \otimes B K) \chi, \\
& \dot{\chi}=(I \otimes(A-B K)-\mu \tilde{L} \otimes I) \chi+\mu(\tilde{L} \otimes I) \tilde{x} .
\end{aligned}
$$

By defining $\delta=\tilde{x}-\chi$, we have

$$
\begin{aligned}
& \dot{\tilde{x}}=(I \otimes(A-B K)) \tilde{x}+(I \otimes B K) \delta \\
& \dot{\delta}=(I \otimes A-\mu \tilde{L} \otimes I) \delta
\end{aligned}
$$

Based on [23, Lemma 7], since the eigenvalues $\tilde{\lambda}_{1}, \ldots, \tilde{\lambda}_{N}$ of $\tilde{L}$ have positive real part, we have

$(S \otimes I)(I \otimes A-\mu \tilde{L} \otimes I)\left(S^{-1} \otimes I\right)=I \otimes A-\mu \tilde{\Lambda} \otimes I$

for a non-singular transformation matrix $S$, where (32) is an upper triangular block matrix with $A-\mu \tilde{\lambda}_{i} I$ for $i=1, \ldots, N$ on the diagonal blocks and $\tilde{\Lambda}$ is upper triangular matrix with $\tilde{\lambda}_{i}$ for $i=1, \ldots, N$ on the diagonals. Since all eigenvalues of $A$ are in the closed left half complex plane, $A-\mu \tilde{\lambda}_{i} I$ is a stable matrix. It means that all eigenvalues of $I \otimes A-\mu \tilde{L} \otimes I$ have negative real part. Thus, it implies we just need to prove the stability of the following system

$\dot{\tilde{x}}=(I \otimes(A-B K)) \tilde{x}$. 
Because $A-B K$ is Hurwitz, It means that $\tilde{x}$ is asymptotically stable, i.e. $\lim _{t \rightarrow \infty} \tilde{x}_{i} \rightarrow 0$. Thus, it implies $x_{i}-x_{r} \rightarrow 0$ which proves the regulated state synchronization.

Step 2: The proof of arbitrary fast convergence

Firstly, we obtain

$\dot{\theta}=\bar{A}_{F} \theta$

by defining $\theta=\left(\begin{array}{ll}\tilde{x}^{\mathrm{T}} & \delta^{\mathrm{T}}\end{array}\right)^{\mathrm{T}}$, where

$\bar{A}_{F}=\left(\begin{array}{cc}I \otimes(A-B K) & I \otimes B K \\ 0 & I \otimes A-\mu \tilde{L} \otimes I\end{array}\right)$.

Similar to Theorem 1 , and given that $\operatorname{Re}\left(\lambda_{i}(A-B K)\right)<\ln \left(r_{\text {asym }}^{*}\right)$, one obtain

$$
\begin{aligned}
r_{\text {asym }} & =\rho\left(e^{\bar{A}_{F}}\right)=\max _{i}\left(e^{\operatorname{Re}\left(\lambda_{i}\left(\bar{A}_{F}\right)\right.}\right)=\max _{i}\left(e^{\operatorname{Re}\left(\lambda_{i}(A-B K)\right)}, e^{\operatorname{Re}\left(\lambda_{i}(I \otimes A-\mu \tilde{L} \otimes I)\right)}\right) \\
& \leq \max _{i}\left(r_{\text {asym }}^{*}, e^{\operatorname{Re}\left(\lambda_{i}(I \otimes A-\mu \tilde{L} \otimes I)\right)}\right) \\
& \leq \max _{i}\left(r_{\text {asym }}^{*}, \max _{j}\left(e^{\operatorname{Re}\left(\lambda_{i}\left(A-\mu \tilde{\lambda}_{j} I\right)\right)}\right)\right) \\
& \leq \max _{i}\left(r_{\text {asym }}^{*}, e^{\operatorname{Re}\left(\lambda_{i}\left(A-\mu \tilde{\lambda}_{1} I\right)\right)}\right)
\end{aligned}
$$

where $\tilde{\lambda}_{j}(j=1, \ldots N)$ are the eigenvalue of $\tilde{L}$ and $\tilde{\lambda}_{1}$ is the eigenvalue of $\tilde{L}$ with the smallest real part.

Then, by choosing sufficiently large $\mu$, we have $\operatorname{Re}\left(\lambda_{i}\left(A-\mu \tilde{\lambda}_{1} I\right)\right)<\ln \left(r_{\text {asym }}^{*}\right)$. Since we have that all eigenvalue of $A$ are in closed right half plane, we only choose $-\mu \operatorname{Re}\left(\tilde{\lambda}_{1}\right) \leq$ $\ln \left(r_{\text {asym }}^{*}\right)$ so as to $r_{\text {asym }} \leq r_{\text {asym }}^{*}$. It means one can achieve regulated state synchronization with arbitrary fast convergence.

\subsection{Partial-state coupling}

We design the Protocol 4 stated in the next page for MAS with partial-state coupling.

Then, we have the following theorem.

Theorem 4. Consider a MAS consisting of $N$ agents (3) satisfying Assumption 1 and the associated exosystem (9). Let the associated network communication be given by Eq. (12).

Then, the problem of scalable regulated state synchronization with arbitrarily fast convergence as defined in Problem 2 is solvable. In particular, the parameterized linear dynamic Protocol 4

- achieves regulated state synchronization for any $\mu$, and any graph $\mathcal{G} \in \mathbb{G}_{\mathscr{C}}^{N}$ with any $N$.

- for a given desired $r_{\text {asym }}^{*}$, any given graph $\mathscr{G} \in \mathbb{G}_{\mathscr{C}}^{N}$ and any $x_{r}$, by choosing parameter $\mu$ sufficiently large, the protocol achieves convergence rate (for the synchronization error) less than $r_{\text {asym. }}^{*}$.

Proof of Theorem 4. Similar to the proof of Theorem 3, first we prove the state synchronization. Let $\tilde{x}_{i}=x_{i}-x_{r}$ and define

$\hat{x}=\left(\begin{array}{lll}\hat{x}_{1}^{\mathrm{T}} & \cdots & \hat{x}_{N}^{\mathrm{T}}\end{array}\right)^{\mathrm{T}}$ 


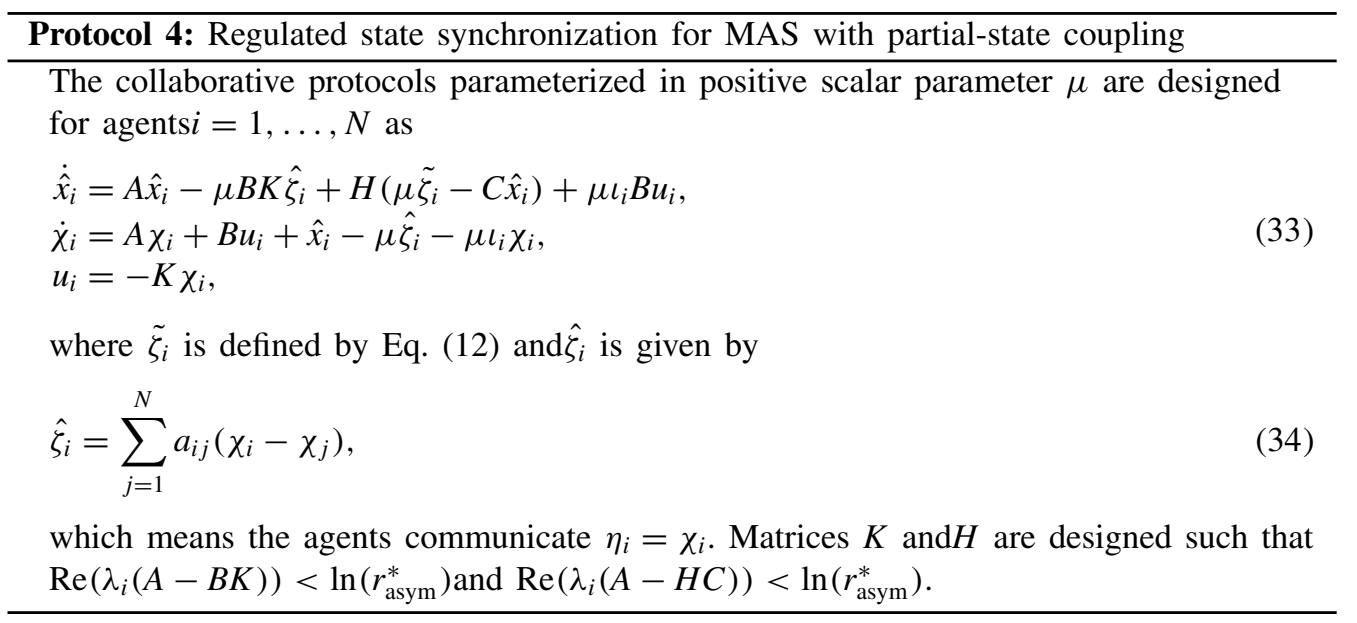

then, we obtain the closed loop system as

$$
\begin{aligned}
& \dot{\tilde{x}}=(I \otimes A) \tilde{x}-(I \otimes B K) \chi \\
& \dot{\hat{x}}=(I \otimes(A-H C)) \hat{x}-(\mu \tilde{L} \otimes B K) \chi+(\mu \tilde{L} \otimes H C) \tilde{x} \\
& \dot{\chi}=(I \otimes(A-B K)-\mu \tilde{L} \otimes I) \chi+\hat{x}
\end{aligned}
$$

By defining $\delta=\tilde{x}-\chi$ and $\bar{\delta}=(\mu \tilde{L} \otimes I) \tilde{x}-\hat{x}$, we obtain

$$
\begin{aligned}
& \dot{\tilde{x}}=(I \otimes(A-B K)) \tilde{x}+(I \otimes B K) \delta \\
& \dot{\delta}=(I \otimes A-\mu \tilde{L} \otimes I) \delta+\bar{\delta} \\
& \dot{\bar{\delta}}=(I \otimes(A-H C)) \bar{\delta}
\end{aligned}
$$

Similar to Theorem 3 , we have that all eigenvalues of $I \otimes A-\mu \tilde{L} \otimes I$ have negative real part and $A-H C$ is Hurwitz stable, it means that we just need to prove the stability of $\dot{\tilde{x}}=$ $(I \otimes(A-B K)) \tilde{x}$. Furthermore, since $A-B K$ is Hurwitz stable, we have $\tilde{x}$ is asymptotically stable, i.e. $\lim _{t \rightarrow \infty} \tilde{x}_{i} \rightarrow 0$. Thus, it implies $x_{i}-x_{r} \rightarrow 0$ which proves the regulated state synchronization result.

Then, we prove the arbitrary fast convergence as the next step of the proof. We have

$\dot{\bar{\theta}}=\bar{A}_{P} \bar{\theta}$

by defining

$\bar{\theta}=\left(\begin{array}{lll}\tilde{x}^{\mathrm{T}} & \delta^{\mathrm{T}} & \bar{\delta}^{\mathrm{T}}\end{array}\right)^{\mathrm{T}}$,

where

$\bar{A}_{P}=\left(\begin{array}{ccc}I \otimes(A-B K) & I \otimes B K & 0 \\ 0 & I \otimes A-\mu \tilde{L} \otimes I & I \\ 0 & 0 & I \otimes(A-H C)\end{array}\right)$. 


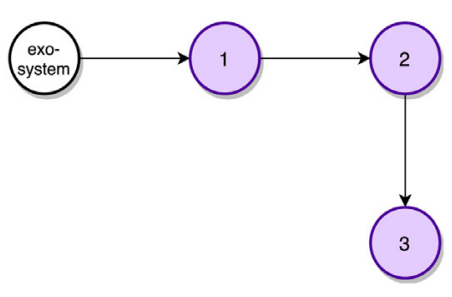

(a) Case I

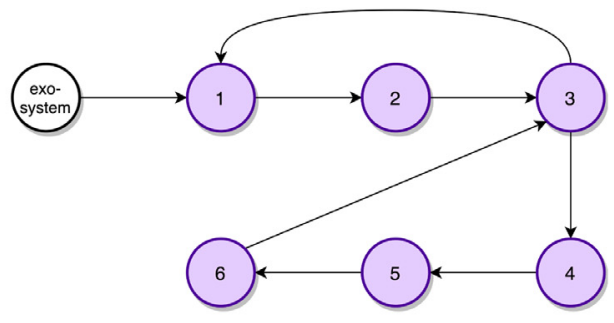

(b) Case II

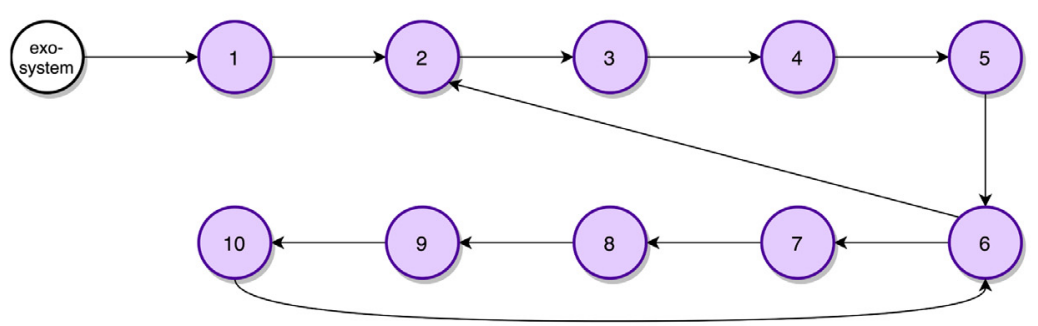

(c) Case III

Fig. 1. The communication topologies.
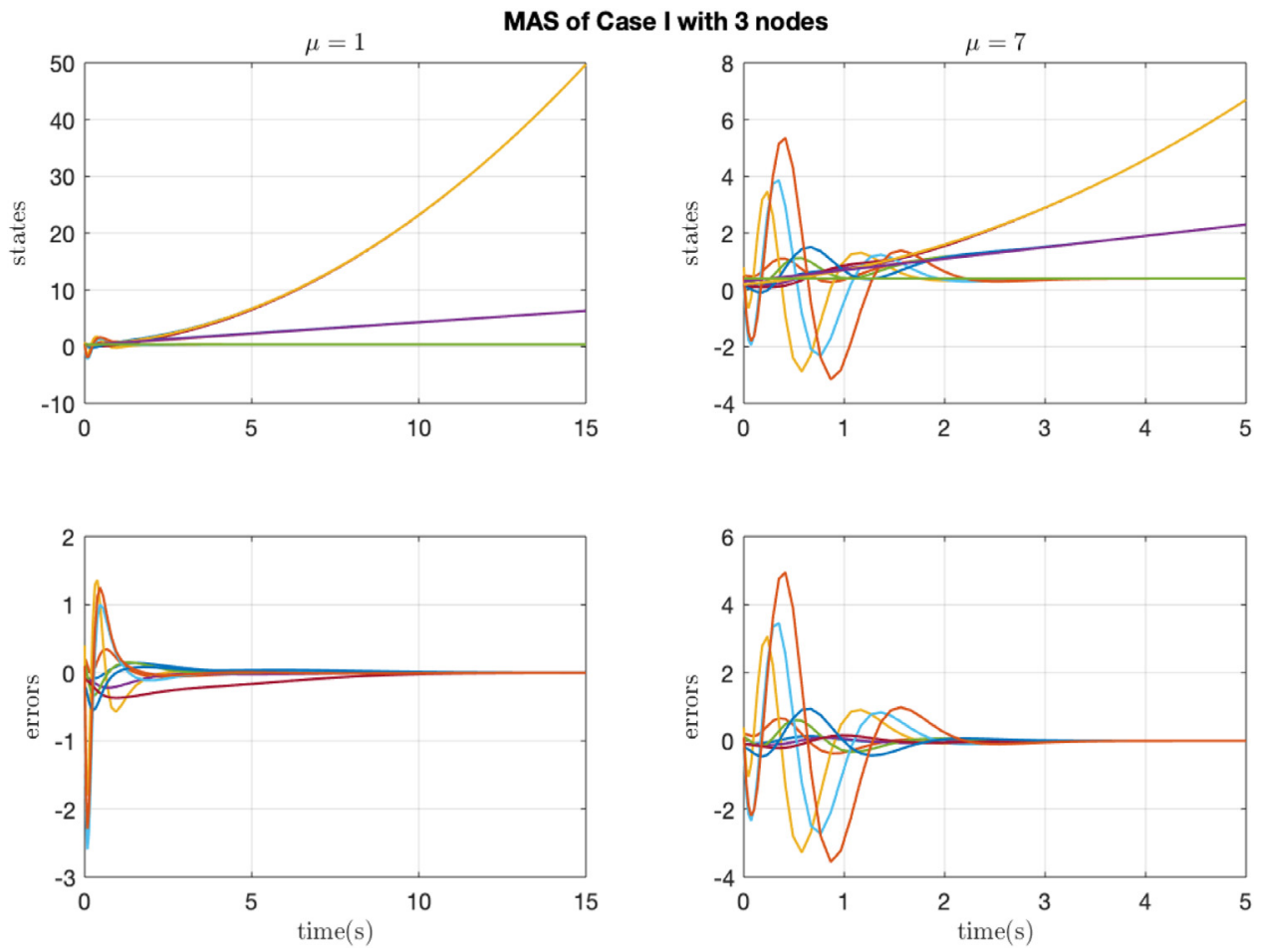

Fig. 2. Regulated state synchronization for MAS with 3 nodes and asymptotic convergence rate $r_{\text {asym }} \geq e^{-5}$ with $\mu=7$. 

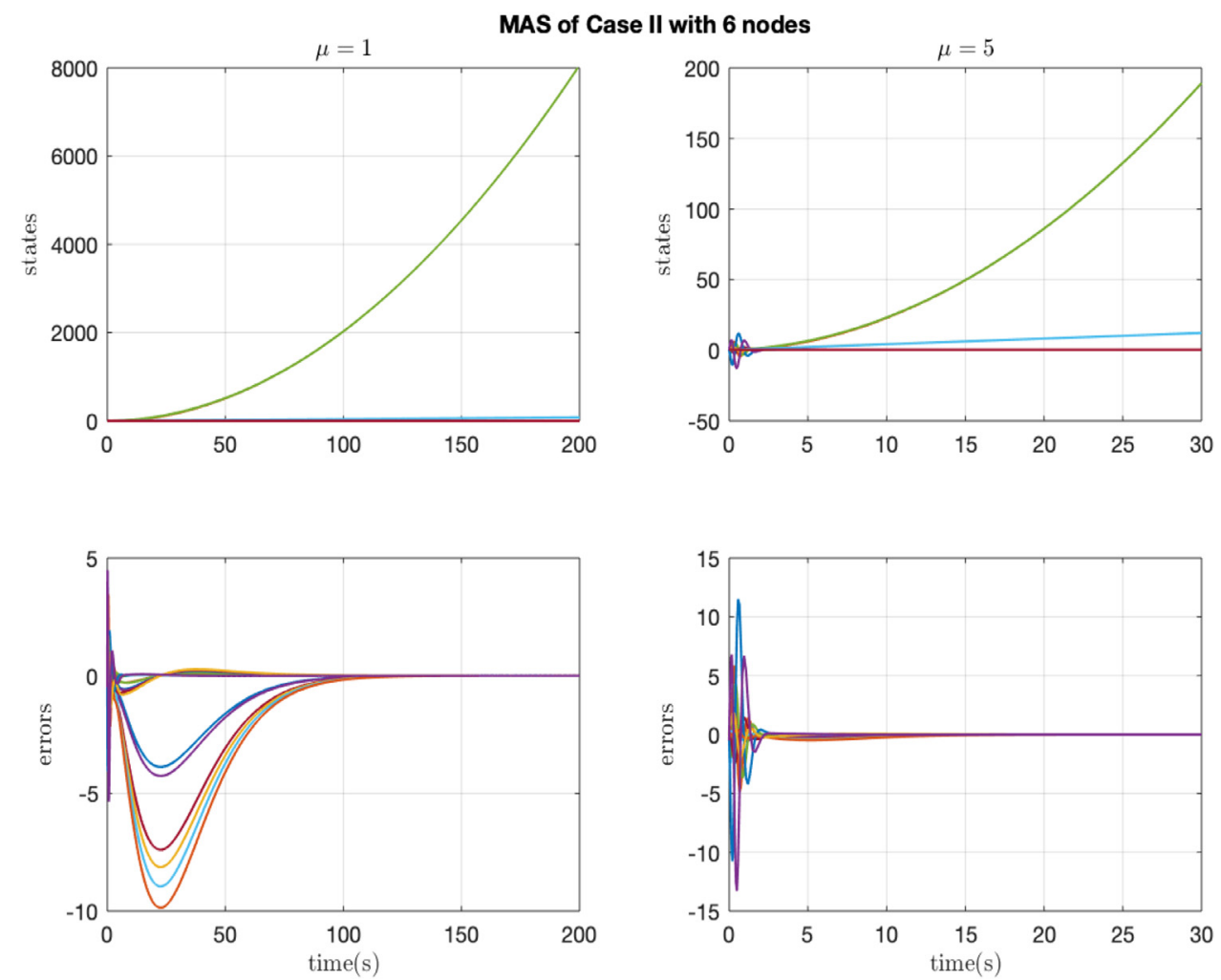

Fig. 3. Regulated state synchronization for MAS with 6 nodes and asymptotic convergence rate $r_{\text {asym }} \geq e^{-5}$ with $\mu=5$.

Similar to Theorem 3, the asymptotic convergence rate of error dynamics would be

$$
\begin{aligned}
r_{\text {asym }} & =\rho\left(e^{\bar{A}_{P}}\right)=\max _{i}\left(e^{\operatorname{Re}\left(\lambda_{i}\left(\bar{A}_{P}\right)\right)}\right) \\
& =\max _{i}\left(e^{\operatorname{Re}\left(\lambda_{i}(A-B K)\right)}, e^{\operatorname{Re}\left(\lambda_{i}(I \otimes A-\mu \tilde{L} \otimes I)\right)}, e^{\operatorname{Re}\left(\lambda_{i}(A-H C)\right)}\right) \\
& \leq \max _{i}\left(r_{\text {asym }}^{*}, e^{\operatorname{Re}\left(\lambda_{i}(I \otimes A-\mu \tilde{L} \otimes I)\right)}\right) \\
& \leq \max _{i}\left(r_{\text {asym }}^{*}, e^{\operatorname{Re}\left(\lambda_{i}(I \otimes A-\mu \tilde{L} \otimes I)\right)}\right) \\
& \leq \max _{i}\left(r_{\text {asym }}^{*}, \max _{j}\left(e^{\operatorname{Re}\left(\lambda_{i}\left(A-\mu \tilde{\lambda}_{j} I\right)\right)}\right)\right) \\
& \leq \max _{i}\left(r_{\text {asym }}^{*}, e^{\operatorname{Re}\left(\lambda_{i}\left(A-\mu \tilde{\lambda}_{1} I\right)\right)}\right)
\end{aligned}
$$

by using the facts $\operatorname{Re}\left(\lambda_{i}(A-B K)\right)<\ln \left(r_{\text {asym }}^{*}\right)$ and $\operatorname{Re}\left(\lambda_{i}(A-H C)\right)<\ln \left(r_{\text {asym }}^{*}\right)$, where $\tilde{\lambda}_{j}$ $(j=1, \ldots N)$ are the eigenvalue of $\tilde{L}$ and $\tilde{\lambda}_{1}$ is the eigenvalue of $\tilde{L}$ with the smallest real part. Then similar to the argument of Theorem 3, we obtain $r_{\text {asym }} \leq r_{\text {asym }}^{*}$ by choosing sufficient large $\mu$. 

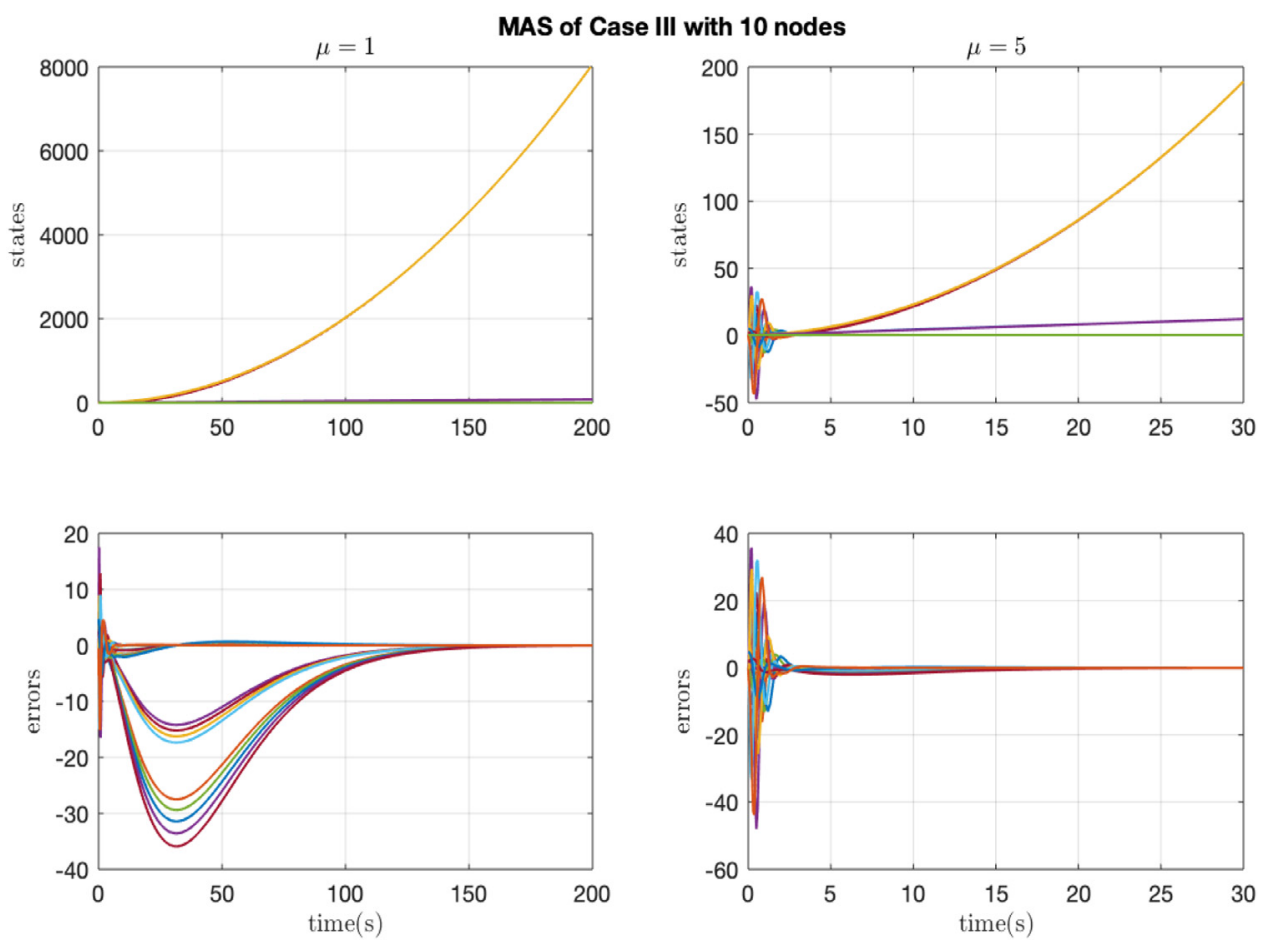

Fig. 4. Regulated state synchronization for MAS with 10 nodes and asymptotic convergence rate $r_{\text {asym }} \geq e^{-5}$ with $\mu=5$.

\section{Simulation results}

In this section, we will illustrate the performance of our parameterized protocols with numerical examples for regulated state synchronization of MASs with partial-state coupling.

Consider agents models (3) and the exosystem (9) with matrices $A, B, C$ and exosystem model (9) with matrices $A, C$, where

$A=\left(\begin{array}{lll}0 & 1 & 0 \\ 0 & 0 & 1 \\ 0 & 0 & 0\end{array}\right), \quad B=\left(\begin{array}{l}0 \\ 0 \\ 1\end{array}\right), \quad C=\left(\begin{array}{lll}1 & 0 & 0\end{array}\right)$.

To achieve asymptotic convergence rate $r_{\text {asym }} \geq e^{-5}$, we choose matrices $K$ and $H$ such that the eigenvalues of $A-B K$ and $A-H C$ are located at $-6,-7$ and -8 . Then we have the following parameterized protocol. Note that the following protocol achieves regulated state synchronization for any $\mu \geq 1$ for any graph.

$$
\begin{aligned}
\dot{\hat{x}}_{i} & =\left(\begin{array}{ccc}
-21 & 1 & 0 \\
-146 & 0 & 1 \\
-336 & 0 & 0
\end{array}\right) \hat{x}_{i}-\mu\left(\begin{array}{ccc}
0 & 0 & 0 \\
0 & 0 & 0 \\
336 & 146 & 21
\end{array}\right) \hat{\zeta}_{i}+\mu\left(\begin{array}{c}
21 \\
146 \\
336
\end{array}\right) \tilde{\zeta}_{i}+\mu \iota_{i}\left(\begin{array}{l}
0 \\
0 \\
1
\end{array}\right) u_{i}, \\
\dot{\chi}_{i} & =\left(\begin{array}{lll}
0 & 1 & 0 \\
0 & 0 & 1 \\
0 & 0 & 0
\end{array}\right) \chi_{i}+\left(\begin{array}{l}
0 \\
0 \\
1
\end{array}\right) u_{i}+\hat{x}_{i}-\mu \hat{\zeta}_{i}-\mu \iota_{i} \chi_{i}, \\
u_{i} & =-\left(\begin{array}{lll}
336 & 146 & 21
\end{array}\right) \chi_{i},
\end{aligned}
$$


To show the scalability of our protocols we choose three different MAS with different communication networks and different number of agents. In all the following cases, we illustrate the results for $\mu=1$. Then, to achieve asymptotic convergence rate $r_{\text {asym }} \geq e^{-5}$, we increase the value of $\mu$ to get $r_{\text {asym }} \geq e^{-5}$.

Case I: Consider a MAS consisting of 3 agents with agent models $(A, B, C)$ and directed communication topology shown in Fig. 1a. Fig. 2 shows the simulation results for $\mu=1$ and $\mu=7$.

Case II: Next, consider a MAS consisting of 6 agents with agent models $(A, B, C)$ and directed communication topology shown in Fig. 1b. Fig. 3 shows the simulation results for $\mu=1$. In this case also to achieve $r_{\text {asym }} \geq e^{-5}$ we increase the value of $\mu$ to 5 .

Case III: Finally, consider a MAS consisting of 10 agents with agent models $(A, B, C)$ and directed communication topology shown in Fig. 1c. Like the previous cases, first we show the simulation results for $\mu=1$. Then, we obtain the simulation results with convergence rate $r_{\text {asym }} \geq e^{-5}$ by choosing the value of $\mu$ equal to 5 . The results are shown in Fig. 4 .

From the simulation results, we observe that our one-shot-design Eq. (37) achieves regulated state synchronization for any graph with any number of agents. Moreover, we can achieve any arbitrarily fast convergence by tuning design parameter $\mu$.

\section{Conclusion}

In this paper, we studied state and regulated state synchronization with arbitrary fast convergence for homogeneous networks of MAS. A scalable linear collaborative dynamic protocol, parameterized in scalar $\mu$ was proposed such that one could achieve any desired given convergence rate by suitably choosing parameter $\mu$. It should be emphasized that the proposed protocols were designed solely based on agent models that is despite all the existing results, our design methodology was scale-free so that we did not need any information about the communication network such as bounds on the spectrum of the associated Laplacian matrix and the number of agents. As the future work, we aim to extend the scale-free protocol designs proposed in this paper to heterogeneous MAS to achieve output synchronization with desired performance. Besides, We would like to extend the results of this paper for homogeneous MAS in presence of communication delays.

\section{Declaration of Competing Interest}

The authors declare that they have no known competing financial interests or personal relationships that could have appeared to influence the work reported in this paper.

\section{References}

[1] H. Bai, M. Arcak, J. Wen, Cooperative Control Design: A Systematic, Passivity-Based Approach, Communications and Control Engineering, Springer Verlag, 2011.

[2] M. Mesbahi, M. Egerstedt, Graph Theoretic Methods in Multiagent Networks, Princeton University Press, Princeton, 2010.

[3] W. Ren, Y.C. Cao, Distributed Coordination of Multi-Agent Networks, Communications and Control Engineering, Springer-Verlag, London, 2011.

[4] C.W. Wu, Synchronization in complex networks of nonlinear dynamical systems, World Scientific Publishing Company, Singapore, 2007.

[5] F. Bullo, Lectures on Network Systems, Kindle Direct Publishing, 2019.

[6] L. Kocarev, Consensus and Synchronization in Complex Networks, Springer, Berlin, 2013. 
[7] S. Stüdli, M.M. Seron, R.H. Middleton, Vehicular platoons in cyclic interconnections with constant inter-vehicle spacing, IFAC-PapersOnLine 50 (1) (2017) 2511-2516.

[8] E. Tegling, B. Bamieh, H. Sandberg, Localized high-order consensus destabilizes large-scale networks, in: Proceedings of the American Control Conference, Philadelphia, PA, 2019, pp. 760-765.

[9] E. Tegling, R.H. Middleton, M.M. Seron, Scalability and fragility in bounded-degree consensus networks, IFACPapersOnLine 52 (20) (2019) 85-90.

[10] Z. Liu, A. Saberi, A.A. Stoorvogel, D. Nojavanzadeh, Global and semi-global regulated state synchronization for homogeneous networks of non-introspective agents in presence of input saturation, in: Proceedings of the 58th CDC, Nice, France, 2019, pp. 7307-7312.

[11] Z. Liu, A. Saberi, A.A. Stoorvogel, D. Nojavanzadeh, $H_{\infty}$ Almost state synchronization for homogeneous networks of non-introspective agents: a scale-free protocol design, Automatica 122 (2020) 109276.

[12] L. Xiao, S. Boyd, Fast linear iterations for distributed averaging, Syst. Control Lett. 53 (1) (2004) 65-78.

[13] A. Stanoev, D. Smilkov, Consensus theory in networked systems, in: L. Kocarev (Ed.), Consensus and Synchronization in Complex Networks, Spinger-Verlag, Berlin, 2013, pp. 1-22.

[14] K. You, L. Xie, Network topology and communication data rate for consensusability of discrete-time multi-agent systems, IEEE Trans. Autom. Control 56 (10) (2011) 2262-2275.

[15] E. Tegling, Fundamental limitations of distributed feedback control in large-scale networks, KTH Royal Institute of Technology, 2018 Ph.D. thesis.

[16] C. Godsil, G. Royle, Algebraic Graph Theory, Graduate Texts in Mathematics, 207, Springer-Verlag, New York, 2001.

[17] R. Horn, C.R. Johnson, Topics in Matrix Analysis, Cambridge University Press, Cambridge, 1991.

[18] X. Shao, D. Ye, Fuzzy adaptive event-triggered secure control for stochastic nonlinear high-order MASs subject to dos attacks and actuator faults, IEEE Trans. Fuzzy Syst. (2021), doi:10.1109/TFUZZ.2020.3028657.

[19] D. Ye, M.-M. Chen, H.J. Yang, Distributed adaptive event-triggered fault-tolerant consensus of multi-agent systems with general linear dynamics, IEEE Trans. Cybern. 49 (3) (2019) 757-767.

[20] T.Y. Zhang, D. Ye, X. Zhao, Completely event-triggered consensus for multi-agent systems with directed switching topologies, IEEE Trans. Cybern. (2021). Early Access. doi: 10.1109/TCYB.2021.3052988.

[21] W. Ao, T. Ma, R.V. Sanchez, H. Gan, Finite-time and fixed-time impulsive synchronization of chaotic systems, J. Frankl. Inst. 357 (16) (2020) 11545-11557.

[22] Y. Yang, D. Xu, T. Ma, X. Su, Adaptive cooperative terminal sliding mode control for distributed energy storage systems, IEEE Trans. Circuits Syst. I Regul. Pap. 68 (1) (2021) 434-443.

[23] H.F. Grip, T. Yang, A. Saberi, A.A. Stoorvogel, Output synchronization for heterogeneous networks of non-introspective agents, Automatica 48 (10) (2012) 2444-2453. 